fundlim, October 28, The fundamental limit on the rate of quantum dynamics:
2018 the unified bound is tight

Lev B. Levitin and Tommaso Toffoli

The question of how fast a quantum state can evolve has attracted a considerable attention in connection with quantum measurement, metrology, and information processing. Since only orthogonal states can be unambiguously distinguished, a transition from a state to an orthogonal one can be taken as the elementary step of a computational process. ${ }^{1}$ Therefore, such a transition can be interpreted as the operation of "flipping a qubit", and the number of orthogonal states visited by the system per unit time can be viewed as the maximum rate of operation.

A lower bound on the orthogonalization time, based on the energy spread $\Delta E$, was found by Mandelstam and Tamm. ${ }^{2}$ Another bound, based on the average energy $E$, was established by Margolus and Levitin. ${ }^{3}$ The bounds coincide, and can be exactly attained by certain initial states if $\Delta E=E$. However, the problem remained open of what the situation is when $\Delta E \neq E$.

Here we consider the unified bound that takes into account both $\Delta E$ and $E$. We prove that there exist no initial states that saturate the bound if $\Delta E \neq E$. However, the bound remains tight: for any given values of $\Delta E$ and $E$, there exists a oneparameter family of initial states that can approach the bound arbitrarily close when the parameter approaches its limit value. The relation between the largest energy level, the average energy, and the orthogonalization time is also discussed. These results establish the fundamental quantum limit on the rate of operation of any information-processing system.

Starting with the classical result of Mandelstam and Tamm, ${ }^{2}$ it was later shown by Fleming, ${ }^{4}$ Anandan and Aharonov, ${ }^{5}$ and Vaidman ${ }^{6}$ that the minimum time $\tau$ required for arriving to an orthogonal state is bounded by

$$
\tau \geq h / 4 \Delta E
$$

where $(\Delta E)^{2}=\left\langle\psi\left|H^{2}\right| \psi\right\rangle-(\langle\psi|H| \psi\rangle)^{2}, H$ is the Hamiltonian, and $|\psi\rangle$ the wavefunction of the system. A different bound was obtained in, ${ }^{3}$ namely,

$$
\tau \geq h / 4 E .
$$

Here, $E=\langle\psi|H| \psi\rangle$ is the quantum-mechanical average energy of the system (the energy of the ground state is taken to be zero). Both bounds (1) and (2) are tight, and achieved for a quantum state such that $\Delta E=E$.

Since then, a vast literature has been devoted to various aspects of this problem. In particular, inequality (2) has been proved for mixed states and for composite systems both in separable and in entangled states (e.g., Giovannetti et al., ${ }^{7,8}$ Zander et $a .^{9}$ ). Bound (2) obtained for an isolated system has been generalized to a system driven by an external Hamiltonian (a "quantum gate") in. ${ }^{10,11}$ Various derivations of (1) and (2) (e.g., $\left.{ }^{12-14}\right)$, bounds based on energy-distribution moments, ${ }^{15}$ more general problems of time-optimal quantum evolution, ${ }^{13,16-24}$ and the ultimate limits of computation ${ }^{25,26}$ have been considered.

However, what remained unnoticed is the paradoxical situation of the existence of two bounds based on two different characteristics of the quantum state, seemingly independent of one another. Since the average energy $E$ and the energy uncertainty $\Delta E$ play the most determinative role in quantum evolution, it is important to have a unified bound that would take into account both of these characteristics.
In all known cases where bounds (1) and (2) can be exactly attained, the ratio $\alpha=\frac{\Delta E}{E}$ equals 1. A question arises: what happens if $\alpha \neq 1$ ? Some authors just assumed without justification that the minimum orthogonalization time is

$$
\tau_{\min }=\max \left(\frac{h}{4 E}, \frac{h}{4 \Delta E}\right)=\frac{h}{2(E+\Delta E-|E-\Delta E|} .
$$

In fact, the situation is not so simple. Bound (3), indeed, can only be achieved for $\alpha=1$. However, this bound remains tight when $\alpha \neq 1$ as well, though in this case it is only asymptotically attainable.

Theorem 1 Under the assumption that the smallest (ground) energy of a quantum system is zero,

1. The only state that attains bound (1) is the two-level state

$$
|\psi\rangle=\frac{1}{\sqrt{2}}\left(\left|\psi_{0}\right\rangle+\left|\psi_{1}\right\rangle\right),
$$

where $H\left|\psi_{k}\right\rangle=k E_{1}$ for $k=0,1$;

2. The only state that attains bound (2) is likewise state (4). State (4) is unique up to degeneracy of the energy level $E_{1}$ and arbitrary phase factors for $\left|\psi_{0}\right\rangle$ and $\left|\psi_{1}\right\rangle$.

Proof. To prove the first statement we shall use the trigonometric inequality

$$
\cos x \geq 1-\frac{4}{\pi^{2}} x \sin x-\frac{2}{\pi^{2}} x^{2},
$$

which is valid for any real $x$. Note that (5) turns into an equality iff $x=0$ or $x= \pm \pi$.

Let the initial state be

$$
|\psi(0)\rangle=\sum_{n=0}^{\infty} c_{n}\left|E_{n}\right\rangle,
$$

where the $\left|E_{n}\right\rangle$ are energy eigenstates of the system and $\sum_{n=0}^{\infty}\left|c_{n}\right|^{2}=1$. Then

$$
\begin{aligned}
|S(t)|^{2} & =|\langle\psi(0) \mid \psi(t)\rangle|^{2} \\
& =\sum_{n, n^{\prime}=0}^{\infty}\left|c_{n}\right|^{2}\left|c_{n^{\prime}}\right|^{2} e^{-i \frac{E_{n}-E_{n^{\prime}}}{\hbar / t}} \\
& =\sum_{n, n^{\prime}=0}^{\infty}\left|c_{n}\right|^{2}\left|c_{n^{\prime}}\right|^{2} \cos \frac{E_{n}-E_{n^{\prime}}}{\hbar / t}
\end{aligned}
$$

Using inequality (5), we obtain

$$
\begin{aligned}
|S(t)|^{2} \geq 1 & -\frac{4}{\pi^{2}} \sum_{n, n^{\prime}=0}^{\infty}\left|c_{n}\right|^{2}\left|c_{n^{\prime}}\right|^{2} \frac{E_{n}-E_{n^{\prime}}}{\hbar / t} \sin \frac{E_{n}-E_{n^{\prime}}}{\hbar / t} \\
& -\frac{2}{\pi^{2}} \sum_{n, n^{\prime}=0}^{\infty}\left|c_{n}\right|^{2}\left|c_{n^{\prime}}\right|^{2}\left(\frac{E_{n}-E_{n^{\prime}}}{\hbar / t}\right)^{2} \\
= & 1+\frac{4 t}{\pi^{2}} \frac{d|S(t)|^{2}}{d t}-\frac{1}{\pi^{2}}\left(\frac{\Delta E}{\hbar / 2 t}\right)^{2} .
\end{aligned}
$$

Since $|S(t)|^{2} \geq 0$, it follows that $\frac{d|S(t)|^{2}}{d t}=0$ whenever $S(t)=$ 0 . Thus, at a time $\tau$ such that $S(\tau)=0$, the second term in (8) vanishes, and we obtain

$$
0 \geq 1-\frac{4 \tau^{2}}{\pi^{2} \hbar^{2}}(\Delta E)^{2}
$$


which yields inequality (11); this is just another way to derive that bound. However, for (9) to turn into an equality, it is necessary that inequality (5) turn into an equality for every term of the double summation (7). Hence,

$$
\text { either } \quad x_{n n^{\prime}}=\frac{E_{n}-E_{n^{\prime}}}{\hbar / \tau}=0 \quad \text { or } \quad x_{n n^{\prime}}=\frac{E_{n}-E_{n^{\prime}}}{\hbar / \tau}= \pm \pi
$$

for all $n, n^{\prime}$ such that $c_{n} \neq 0, c_{n^{\prime}} \neq 0$. It follows that, to attain bound (1), $|\psi(0)\rangle$ must be a superposition of only two energy eigenstates with energies $E_{0}=0$ and $E_{1}$.

To prove the second statement, we repeat briefly the derivation given in. ${ }^{3}$ This time we use the trigonometric inequality

$$
\cos x \geq 1-\frac{2}{\pi}(x+\sin x)
$$

valid for all $x \geq 0$. Again, (11) turns into an inequality only for $x=0$ or $x=\pi$.

Then

$$
\begin{aligned}
\operatorname{Re} S(t) & =\sum_{n=0}^{\infty}\left|c_{n}\right|^{2} \cos \frac{E_{n} t}{\hbar} \\
& \geq \sum_{n=0}^{\infty}\left|c_{n}\right|^{2}\left[1-\frac{2}{\pi}\left(\frac{E_{n} t}{\hbar}+\sin \frac{E_{n} t}{\hbar}\right)\right] \\
& =1-\frac{2 E t}{\pi \hbar}+\frac{2}{\pi} \operatorname{Im} S(t) .
\end{aligned}
$$

At time $\tau, \operatorname{Re} S(\tau)=\operatorname{Im} S(\tau)=0$. Hence $0 \geq 1-\frac{2 E t}{\pi \hbar}$, which results in bound (2). However, to actually attain this bound, inequality (11) must become an equality for every term of the sum (12); that is, for any $n$,

$$
\text { either } \quad x_{n}=\frac{E_{n} t}{\hbar}=0 \quad \text { or } \quad x_{n}=\frac{E_{n} t}{\hbar}=\pi ;
$$

This is possible iff $|\psi(0)\rangle$ has the form (4).

Let us show now that no mixed state can attain bound (3). Consider an initial mixed state density matrix $\rho(0)$ with spectral decomposition

$$
\rho(0)=\sum_{i} \lambda_{i}\left|\psi^{(i)}(0)\right\rangle\left\langle\psi^{(i)}(0)\right|,
$$

where $\lambda_{i}>0, \sum_{i} \lambda_{i}=1$, and $\left\langle\psi^{(i)}(0) \mid \psi^{(j)}(0)\right\rangle=\delta_{i j}$. At time $t$, the density matrix becomes

$$
\rho(t)=\sum_{i} \lambda_{i}\left|\psi^{(i)}(t)\right\rangle\left\langle\psi^{(i)}(t)\right|
$$

Hence, if $\tau$ is the orthogonalization time, then

$$
\operatorname{Tr}[\rho(0) \rho(\tau)]=\sum_{i} \sum_{j} \lambda_{i} \lambda_{j}\left|\left\langle\psi^{(i)}(0) \mid \psi^{(j)}(t)\right\rangle\right|^{2}=0 .
$$

Note that all terms in the above sum are non-negative, and therefore all of them must be zero to satisfy (14). As shown above, terms $\left\langle\psi^{(i)}(0) \mid \psi^{(i)}(\tau)\right\rangle=0$, for $\tau$ given by (3), iff each $\left|\psi^{(i)}(0)\right\rangle$ has the form (4).

Consider two functions from spectral decomposition (13)

$$
\begin{aligned}
& \left|\psi^{(i)}(0)\right\rangle=\frac{1}{\sqrt{2}}\left(\left|\psi_{0}\right\rangle+\left|\psi_{1}^{(i)}(0)\right\rangle\right) \text { and } \\
& \left|\psi^{(j)}(0)\right\rangle=\frac{1}{\sqrt{2}}\left(\left|\psi_{0}\right\rangle+a\left|\psi_{1}^{(i)}(0)\right\rangle+b\left|\psi_{1}^{(j)}(0)\right\rangle\right),
\end{aligned}
$$

where $\left\langle\psi^{(i)}(0) \mid \psi^{(j)}(0)\right\rangle=0$ and $|a|^{2}+|b|^{2}=1$ (the zero-energy state is nondegenerate). Since

$$
\left\langle\psi^{(i)}(0) \mid \psi^{(j)}(0)\right\rangle=\frac{1}{2}\left(\left\langle\psi_{0} \mid \psi_{0}\right\rangle+a\left\langle\psi_{1}^{(i)}(0) \mid \psi_{1}^{(i)}(0)\right\rangle\right)=0,
$$

it follows that $a=-1$ and $\left|\psi^{(j)}(0)\right\rangle=\frac{1}{\sqrt{2}}\left(|\psi(0)\rangle-\left|\psi_{1}^{(i)}(0)\right\rangle\right)$. But then

$$
\left\langle\psi^{(i)}(0) \mid \psi^{(j)}(\tau)\right\rangle=\frac{1}{2}\left(\left\langle\psi_{0} \mid \psi_{0}\right\rangle-\left\langle\psi_{1}^{(i)}(0) \mid \psi_{1}^{(i)}(\tau)\right\rangle\right)=\frac{1}{2} \neq 0 .
$$

Hence, equality (14) cannot be satisfied for $\tau$ given by (3). Thus, this bound is not attainable by a mixed state.

For pure states, results of Theorem 1 also follow from the analyses presented in ${ }^{12}$ and. ${ }^{15}$

Theorem 1 shows that bounds (1) and (2) can only be attained by a state for which $\alpha=\frac{\Delta E}{E}=1$. Such a state is unique up to degeneracy of the energy level $E_{1}$ and arbitrary phase factors for $\left|\psi_{0}\right\rangle$ and $\left|\psi_{1}\right\rangle$. It follows from that theorem that there exists no initial state $|\psi(0)\rangle$ with $\alpha \neq 1$ that would attain bound (3), i.e., no state can evolve into an orthogonal state in the minimum time given by (1) or (2). The question to be answered is how close it is possible to approach the unified bound (3). Let us rewrite (3) in a different form,

$$
\tau_{\min }=\max \left(\frac{h}{4 E}, \frac{h}{4 \Delta E}\right)=\frac{h\left(1+e^{|\ln \alpha|}\right)}{4 E(1+\alpha)} .
$$

Theorem 2 For any $\alpha \neq 1$ and any $\varepsilon>0$, there exists a state $|\psi(0)\rangle$ such that $\langle\psi(0) \mid \psi(\tau)\rangle=0$ at time

$$
\tau \leq \frac{h\left(1+e^{|\ln \alpha|}\right)}{4 E(1+\alpha)}(1+\varepsilon) .
$$

Proof. We will show that in both cases, $\alpha<1$ and $\alpha>1$, there exist families of initial states that approach limit (16) arbitrarily close.

1. Let $\alpha<1$. Consider a family of states

$$
|\psi(0)\rangle=c_{0}|0\rangle+c_{1}\left|E_{1}\right\rangle+c_{2}\left|E_{2}\right\rangle .
$$

Denote $\left|c_{i}\right|^{2}=p_{i}$, and introduce dimensionless variables

$$
x_{i}(t)=2 \pi E_{i} \frac{t}{h}, \quad i=0,1,2
$$

(the rotation angles of the state vectors $\left|E_{i}\right\rangle$ ). Then $S(\tau)=$ $\langle\psi(0) \mid \psi(\tau)\rangle=0$ iff $x_{i}(\tau)=x_{i}$ such that

$$
p_{1} \sin x_{1}+p_{2} \sin x_{2}=0
$$

and

$$
p_{0}+p_{1} \cos x_{1}+p_{2} \cos x_{2}=0 \text {. }
$$

Of course,

$$
p_{0}+p_{1}+p_{2}=1 \text {. }
$$

Let $0<p_{0}<<1$ be a parameter of the family of states (18) (one cannot set $p_{0}=0$, since that would change the ground energy level). When $p_{0}$ is small, the values of $x_{1}$ and $x_{2}$ differ almost exactly by $\pi$. Let

$$
x_{2}=\pi+x_{1}-\delta \sin x_{1}, \quad \text { with } \delta<<1 .
$$

Then, from (20),

$$
p_{1}=p_{2}\left[1-\delta \cos x_{1}+\mathrm{O}\left(\delta^{2}\right)\right] .
$$

Substituting (24) into (21) and (22) we obtain

$$
\begin{aligned}
& p_{1}=\frac{1}{2}-\frac{\delta}{4}\left(1+\cos x_{1}\right)+\mathrm{O}\left(\delta^{2}\right), \\
& p_{2}=\frac{1}{2}-\frac{\delta}{4}\left(1-\cos x_{1}\right)+\mathrm{O}\left(\delta^{2}\right), \\
& p_{0}=\frac{\delta}{2}+\mathrm{O}\left(\delta^{2}\right) .
\end{aligned}
$$


Using (23) and (25), we can calculate the average value $\langle x\rangle=$ $\frac{2 \pi \tau}{h} E$, namely,

$$
\begin{aligned}
\langle x\rangle & =p_{1} x_{1}+p_{2} x_{2} \\
& =\frac{\pi}{2}+x_{1}-\frac{\delta}{4}\left[2 x_{1}-2 \sin x_{1}+\pi\left(1-\cos x_{1}\right)\right]+\mathrm{O}\left(\delta^{2}\right) .
\end{aligned}
$$

The standard deviation $\Delta x=\frac{2 \pi \tau}{h} \Delta E$ is obtained as

$$
\begin{aligned}
\Delta x & =\sqrt{p_{1} x_{1}^{2}+p_{2} x_{2}^{2}-\left(p_{1} x_{1}+p_{2} x_{2}\right)^{2}} \\
& =\frac{\pi}{2}+\frac{\delta}{2 \pi}\left[x_{1}\left(\pi+x_{1}\right)-\pi \sin x_{1}\right]+\mathrm{O}\left(\delta^{2}\right) .
\end{aligned}
$$

On the other hand,

$$
\alpha=\frac{\Delta E}{E}=\frac{\Delta x}{\langle x\rangle}=\frac{\pi}{\pi+2 x_{1}}+\mathrm{O}(\delta) .
$$

Hence,

$$
x_{1}=\frac{\pi}{2}\left(\frac{1}{\alpha}-1\right)+\mathrm{O}(\delta) .
$$

Substituting (29) into (27) and taking into account the last expression from (25) yields

$$
\Delta x=\frac{\pi}{2}+p_{0}\left[\frac{\pi}{4}\left(\frac{1}{\alpha^{2}}-1\right)-\sin \frac{\pi}{2}\left(\frac{1}{\alpha}-1\right)\right]+\mathrm{O}\left(p_{0}^{2}\right),
$$

which results in

$$
\begin{aligned}
\tau & =\frac{h \Delta x}{2 \pi \Delta E} \\
& =\frac{h}{4 \Delta E}\left[1+\frac{p_{0}}{2}\left(\frac{1}{\alpha^{2}}-1-\frac{4}{\pi} \sin 2 \pi\left(\frac{1}{\alpha}-1\right)\right)+\mathrm{O}\left(p_{0}^{2}\right)\right] .
\end{aligned}
$$

Finally, choosing

$$
p_{0}<2 \varepsilon\left[\frac{1}{\alpha^{2}}-1-\frac{2}{\pi} \sin \frac{\pi}{2}\left(\frac{1}{\alpha}-1\right)\right]^{-1},
$$

we obtain

$$
\frac{h}{4 \Delta E}<\tau \leq \frac{h}{4 \Delta E}(1+\varepsilon) .
$$

2. Let $\alpha>1$. Consider a family of states

$$
|\psi(0)\rangle=c_{0}|0\rangle+c_{1}\left|E_{1}\right\rangle+c_{2 k+1}\left|E_{2 k+1}\right\rangle,
$$

where

$$
\begin{aligned}
& k=1,2, \ldots ; E_{2 k+1}=(2 k+1) E_{1} ;\left|c_{0}\right|^{2}=p_{0}=\frac{1}{2} ; \\
& \left|c_{1}\right|^{2}=p_{1}=\frac{1}{2}\left(1-\frac{\beta}{k^{2}}\right) ;\left|c_{2 k+1}\right|^{2}=p_{2 k+1}=\frac{\beta}{2 k^{2}} .
\end{aligned}
$$

Using the dimensionless variables (19), it is readily seen from (33) and (34) that $S(\tau)=0$ at the least time $\tau$ for which

$x_{1}(\tau)=x_{1}=\frac{2 \pi \tau}{h} E_{1}=\pi$ and $x_{2 k+1}=\frac{2 \pi \tau}{h}(2 k+1) E_{1}=\pi(2 k+1)$.

Then

$$
\langle x\rangle=p_{1} x_{1}+p_{2 k+1} x_{2 k+1}=\frac{\pi}{2}\left(1+\frac{2 \beta}{k}\right)
$$

and

$$
\begin{aligned}
\Delta x & =\sqrt{p_{1} x_{1}^{2}+p_{2 k+1} x_{2 k+1}^{2}-\left(p_{1} x_{1}+p_{2 k+1} x_{2 k+1}\right)^{2}} \\
& =\frac{\pi}{2}\left(1+4 \beta+\frac{2 \beta}{k}\right)+\mathrm{O}\left(\frac{1}{k^{2}}\right) .
\end{aligned}
$$

Hence, $\alpha=\frac{\Delta x}{\langle x\rangle}=1+4 \beta+\mathrm{O}\left(\frac{1}{k}\right)$ and

$$
\beta=\frac{\alpha-1}{4}+\mathrm{O}\left(\frac{1}{k}\right) .
$$

Since $\langle x\rangle=\frac{2 \pi \tau}{h} E$, it follows from (37) and (38) that

$$
\tau=\frac{h}{4 E}\left[1+\frac{\alpha-1}{2 k}+\mathrm{O}\left(\frac{1}{k^{2}}\right)\right] .
$$

Thus, choosing a sufficiently large $k>\frac{\alpha-1}{2 \varepsilon}$ guarantees that

$$
\frac{h}{4 E}<\tau \leq \frac{h}{4 E}(1+\varepsilon) .
$$

This completes the proof.

As graphically illustrated in Fig.1 Theorem 2 means that there are families of states that approach equality in (3) for a given value of $\alpha$, in the limit as a certain parameter approaches zero (for $\alpha<1$ ) or infinity (for $\alpha>1$ ), but no state yields a strict equality in (3) for $\alpha \neq 1$. It turns out that bound (1) is tight whenever $\alpha=\frac{\Delta E}{E} \leq 1$, while (2) is tight whenever $\alpha \geq 1$.

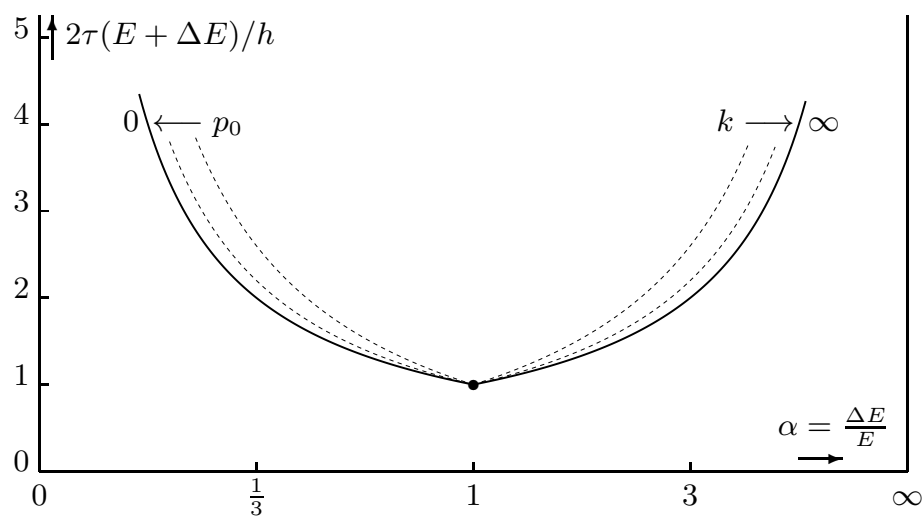

Figure 1: The solid line shows bound (16); the dotted linesparametrized, respectively, by $p_{0} \rightarrow 0$ and $k \rightarrow \infty$ - show successive approximations to this bound, corresponding to initial states from families (18) and (33).

Another interesting question is the relationship between the maximum energy eigenvalue $E_{\max }$ that contributes to $|\psi(0)\rangle$, the orthogonalization time, $\tau$, and the average energy $E$. Note that determining the minimum $\tau$ for a given $E$ is equivalent to determining the minimum $E$ for a given $\tau$. The next theorem provides simple but useful results.

Theorem 3 Let $\tau$ be the minimum time such that $S(\tau)=$ $\langle\psi(0) \mid \psi(\tau)\rangle=0$. Then there exist a state $|\psi(0)\rangle$ and a set of energy eigenvalues $\left\{E_{n}\right\}$ of all energy eigenfunctions that contribute to $\left|\psi_{0}\right\rangle$ such that

$$
E_{\max }<\frac{h}{\tau}
$$

and

$$
\frac{E_{\max }}{4} \leq E \leq \frac{E_{\max }}{2}
$$

Proof. Let

$$
S(\tau)=\sum_{n=0}^{\infty}\left|c_{n}\right|^{2} e^{-2 \pi i E_{n} \tau / h}=0 .
$$

Suppose $E_{k} \geq \frac{h}{\tau}$. Then $E_{k}^{\prime}=E_{k}-\frac{h}{\tau} \geq 0$ and $e^{-2 \pi i E_{k} \tau / h}=$ $e^{-2 \pi i E_{k}^{\prime} \tau / h}$. Hence, replacing $E_{k}$ by $E_{k}^{\prime}$ in (43) will not affect the equality. Thus, the same ortogonalization time $\tau$ can be achieved with smaller average energy $E^{\prime}=E-\left|c_{k}\right|^{2} h / \tau$. This proves inequality (41).

Now, let $E_{\max }$ be the largest energy of the energy eigenfunctions that contribute to $|\psi(0)\rangle$, and let the average energy be $E^{(1)}$. Obviously, the validity of (43) will not be affected if we replace all energy levels $E_{n}$ by $E_{\max }-E_{n}$. Then the average energy will become $E^{(2)}=E_{\max }-E^{(1)}$. Since $E$ can be chosen 
as $\min \left(E^{(1)}, E^{(2)}\right)$, this proves that $E \leq E_{\max } / 2$. Also, substituting (2) into (41) yields $E_{\max } / 4 \leq E$. Thus, (42) is proved.

Theorem 3 allows us to restrict our attention to states that satisfy (41) and (42) for further analysis of the orthogonalization time $\tau$.

Theorem 4 Let $E_{\max }$ be the maximum energy of all energy eigenfunctions that contribute to $|\psi(0)\rangle$. Then the minimum orthogonalization time $\tau$ obeys the inequality

$$
\tau \geq \frac{h}{2 E_{\max }}
$$

and equality is attained iff $|\psi(0)\rangle$ has the form (4) with $E_{1}=E_{\max }$.

$$
\begin{aligned}
& \text { Proof. Let } \\
& \qquad S(\tau)=\langle\psi(0) \mid \psi(\tau)\rangle=\sum_{n=0}^{m}\left|c_{n}\right|^{2} e^{-2 \pi i E_{n} / h}=0,
\end{aligned}
$$

where $E_{m}=E_{\max }$. Then, if $\tau<\frac{h}{2 E_{\max }}$, all terms in the above sum except the zeroth have strictly positive imaginary parts - which violates equality (45). Similarly, if $\tau=\frac{h}{2 E_{\max }}$, then (45) is satisfied iff $|\psi(0)\rangle$ is of form (4) with $E_{1}=E_{\max }$.

The equality case in (44) had been considered in. ${ }^{14}$

Some authors (e.g., ${ }^{14}$ ) prefer to write expressions (2) and (44) as $\tau \geq h / 4\left(E-E_{0}\right)$ and $\tau \geq h / 2\left(E_{\max }-E_{0}\right)$, arguing that a constant shift of the energy spectrum affects only the overall time-dependent phase of the wavefunction. In our opinion, however, it is just this "freedom of shift" that allows one to set to zero the energy of the ground state even if the smallest eigenvalue of the Hamiltonian is not zero (just as in the case of the hydrogen atom).

The authors wish to thank Prof. D. Brody (Imperial College, London) for a useful discussion.

\section{List of references}

${ }^{1}$ Levitin, LB, "Physical limitations of rate, depth and minimum energy in information processing," Int. J Theor. Phys. 21 (1982), 299-309.

2 Mandelstam, L, and I TAmm, "The uncertainty relation between energy and time in non-relativistic quantum mechanics," $J$ Physics 9, Moscow (1945), 249-254.

3 Margolus N, and LB Levitin, "The maximum speed of dynamical evolution," Physica D 120 (1998), 188-195.

${ }^{4}$ Fleming, GN, "A unitary bound on the evolution of nonstationary states," Nuovo Cimento A 16 (1973), 232-240.

5 Anandan, J, and Y Aharonov, "Geometry of quantum evolution," Phys. Rev. Lett. 65 (1990), 1967-1760.

${ }^{6}$ Vaidman, Lev "Minimum time for the evolution to an orthogonal quantum state," Am. J Phys. 60 1992, 182-183.

7 Giovannetti, V, S Lloyd, and L Maccone, "Quantum limits to dynamical evolution," Phys. Rev. A 67052109 (2003), 8pp.

${ }^{8}$ Giovannetti, V, S Lloyd, and L Maccone, "The role of entanglement in dynamical evolution," Europhys. Lett 62 (2003), 615-621.

${ }^{9}$ Zander, C, AR Plastino, A Plastino, and M Casas, "Entanglement and the speed of evolution of multi-partite quantum systems," J Phys. A 40 (2007), 2861-2872.
10 Levitin, LB, T TofFoli, and Z WAlton, "Operation time of quantum gates," in Quantum Commmunication, Measurement and Computing (J Schapiro and O Hirota, eds.), Rinton 2003, 457459 .

${ }^{11}$ Levitin, L, T Toffoli, and Z Walton, "Maximum speed of quantum gate operation," Int. J Theor. Phys. 44 (2005), 965-970.

12 KosińsKI, P, and M ZYCH, "Elementary proof of the bound of the speed of quantum evolution," Phys. Rev. A 73024303 (2006).

13 Andrecut, M, and MK Ali, "Maximum speed of quantum evolution," Int. J Theor. Phys. 43 (2004), 969-974.

${ }^{14}$ Brody, DC, "Elementary derivation of passage times," $J$ Phys. A 36 (2003), 5587-5593.

15 Zielinski, B, and M ZYCH, "Generalization of the MargolusLevitin bound," Phys. Rev. A 74034301 (2006), 3pp.

16 Brody, DC, and DW Hook, "On optimum Hamiltonians for state transformations," J Phys. A 39 (2006), L167-L170.

17 Carlini, A, A Hosoya, T Kolke, and Y Okudaira, "Timeoptimal quantum evolution," PRL 96060503 (2006), 4pp.

18 LuO, S, "How fast can a quantum state evolve into a target state?" Physica D 109 (2004), 1-7.

19 PAті, AK, "Uncertainty relation of Anandan-Aharonov and intelligent states," Phys. Lett. A 262 (1999), 296-301.

20 Pfeifer, P, "How fast can a quantum state change with time?" Phys. Rev. Lett. 70 (1993), 3365-3368.

${ }^{21}$ Carlini, A, A Hosoya, T Kolke, and Y Okudaira, "Time optimal unitary operations," arXiv:quant-ph/06080039v4, 11 April 2007.

22 Borras, A, C Zander, AR Plastino, M Casas, and A Plastino, "Entanglement and the quantum brachistochrone problem," EPL 8130007 (2007), 5pp.

23 Söderholm, J, B Gunnar, T Tedros, and A Trifonov, "States that minimize the evolution time to become an orthogonal state," Phys. Rev. A 59 (1999), 1788-1790.

${ }^{24}$ Uffink, J, "The rate of evolution of a quantum state," Amer. $J$ Physics 61 (1993), 935-938.

${ }^{25}$ LlOyd, S, "Ultimate physical limits to computation," Nature 406 (2000), 1047-1054.

${ }^{26}$ LLOYD, "Computation capacity of the universe," PRL 88237901 (2002). 